This work has been submitted to NECTAR, the Northampton Electronic Collection of Theses and Research.

\title{
Article
}

Title: Cultural basis of social 'deficits' in autism spectrum disorders

Creator: Perepa, P.

DOI: $10.1080 / 08856257.2014 .908024$

Example citation: Perepa, P. (2014) Cultural basis of social 'deficits' in autism spectrum disorders. European Journal of Special Needs Education. 0885-6257. (In Press)

It is advisable to refer to the publisher's version if you intend to cite from this work.

Version: Accepted version

Official URL: http://dx.doi.org/10.1080/08856257.2014.908024

Note: This is an Accepted Manuscript of an article published by Taylor \& Francis in European Journal of Special Needs Education on 29 Apr 2014, available online: http://wwww.tandfonline.com/10.1080/08856257.2014.908024.

$$
\text { http://nectar.northampton.ac.uk/6678/ }
$$

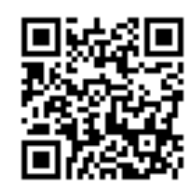




\title{
Cultural basis of social 'deficits' in Autism Spectrum Disorders
}

\author{
Dr. Prithvi Perepa
}

School of Education, University of Northampton, Northampton, UK

Senior Lecturer SEN \& Inclusion, University of Northampton, Park Campus, Boughton Green Road, Northampton, NN2 7AL, email: prithvi.perepa@northampton.ac.uk

This is an Author's Accepted Manuscript of an article published in European Journal of Special Needs Education [date of publication], available online:

http://www.tandfonline.com/10.1080/08856257.2014.908024 


\section{Cultural basis of social 'deficits' in Autism Spectrum Disorders}

There is very little research that specifically looks at how autism spectrum disorders are perceived in various communities. This qualitative research was conducted with parents who had children on the autistic spectrum belonging to four different ethnic communities (White British, Somali, West African and South Asian- 63 in total) and living in the UK. The study found that the importance that the parents give to various social skills varied on the basis of their cultural background and the gender of the parent. This is an important aspect to consider while providing support and services to individuals on the autism spectrum and their family members if the services have to be appropriate for their needs. This consideration would also enable the individuals on the autism spectrum to develop appropriate social skills required within their cultural groups. This is a preliminary study and further research on the topic is required.

Keywords: autism, social skills, parental perceptions, culture, ethnicity

\section{Introduction}

According to some prevalent studies, autism spectrum disorders (ASD) are understood to affect people from all ethnic backgrounds equally (Fombonne et al., 2001). Although diagnostic criteria until recently were underpinned by a ‘triad of impairments’ (Wing, 1988), the disorder was in practice most typically characterised by difficulties in social interactions (Charman \& Stone, 2006). Teaching the 'hidden curriculum' of social skills is considered to be an essential aspect of working with individuals on the autism spectrum.

It is argued that social understanding and social development are most affected by cultural factors and that accepted social norms are subject to considerable cultural variation (Dyches et al., 2005). It is possible therefore that there are differences in how 
a disability is perceived on the basis of the social and cultural milieu of which it is a part (Shakespeare and Watson, 2001; Fletcher \& Navarrete, 2003). In fact there have been academics who have argued that disabilities such as learning difficulties, behaviour, emotional and social difficulties are socially constructed (Gabel, 2004; Hatton, 2004; Lindsay, Pather and Strand, 2006) for precisely the same reasons. It has also been suggested that the system of special education is also culturally bound and that parents from minority ethnic communities can feel disenfranchised when they encounter and access such systems (Zionts et al., 2003; Kalyanpur and Harry, 2004). However, such discussions are still relatively new when it comes to autism; and there is very little research on how parents from different cultural backgrounds interpret the defining social behaviours used to identify autism.

In this exploratory project, parents of children on the autism spectrum from four different cultural backgrounds in the UK were interviewed to discover how they interpreted the same set of social behaviours, all linked to an autism spectrum diagnosis. It was envisaged that such study could contribute to the emerging discussions about the social construct of autism.

\section{Social behaviour and culture}

Culture can be defined as the distinctive way of life of a group, based on similar values, and involving attitudes which influence their behaviour (Kai, Loudon, and Beavan, 2006). One of the main purposes of social competencies is to enable individuals to function fully in their particular social situation. The notion of appropriate social behaviour becomes especially relevant when one faces a different culture to one's 
own, where even well-tuned social skills may not be considered as acceptable or appropriate, since interpretation of social behaviour rests upon knowledge of social customs, rules, and norms for that group (Burton, Kagan, and Clements, 1995). The following section discusses some of the social behaviours in relation to autism.

\section{Differences in social expectations:}

Wilder et al. (2004) and Liu, (2005), for example, found that the level of eye contact provided by individuals from Asian American cultures seem to vary from that of White Americans. The gender of the individuals was also another factor which influenced these differences. Perepa (2013) comments that similar patterns are found in some Nigerian families in the UK, for whom giving direct eye contact was considered rude; conversely, in Western society eye contact is a required behaviour. In fact lack of eye contact is considered as one of the symptoms of the disorder when found in individuals with ASD and is part of many screening tools such as Q-CHAT (Allison et al. 2008). Dyches et al. (2007) argue that when teachers continue teaching children from culturally diverse communities to give eye contact, they are in fact violating the family’s cultural norms.

Similar cultural differences in use of non-verbal communication (Liu, 2005), language (Kim, Kim and Rue, 1997), and achieving of developmental milestones (Daley, 2004) have been reported in various cross-cultural studies. For example, in communities where children are expected to have a passive role, some of the features of autism such as repetitive questioning or echolalia can be viewed as more challenging by the parents (Dyches et al. 2007). These different perceptions could have an impact on whether or not a family considers these behaviours as difficulties or differences that need to be addressed, which behaviours are considered to be more problematic, which 
services the family accesses, and when they do so. Having said that, most of these authors have not focused on parents of children with autism or families from minority ethnic communities; which limits their generalizability to families of children with autism from minority ethnic communities living in multicultural European countries such as the UK.

There is however a growing awareness of the cultural basis of behaviours in the field of autism. Expanding on the idea of a cultural basis for observed behaviour, Wilder et al. (2004) add that a number of behaviours such as tantrums, aggression, lack of normal attachment to family members, poor social-interaction and communication skills, and lack of emotional expression, all have their roots in cultural differences and expectations. They provide the example of Asian-American children who may avoid eye contact with adults, and respond to the teacher's questions by being silent, out of respect for the adults. According to them these behaviours could be interpreted by the school staff, who are unaware of their cultural relevance, as difficulties in the areas of social skills and communication, and hence treated as possible symptoms of ASD. Lee (2011) also suggests that Asian American students’ lack of participation within a classroom could be related to respect for elders. Lee further argues that it is necessary to study cultural differences, as some of the language difficulties (such as understanding idioms) experienced by individuals with autism coming from a minority ethnic community could be related to their level of understanding of English and not autism per se. If teachers are unaware of such differences they could be mislabelling the children or teaching them culturally inappropriate skills. 
In one of the few cross-cultural studies in autism, Matson et al., (2011) did involve family members to research reported features of autism but found that there are no significant differences in the reported social behaviours across these various countries. However, in a follow up study conducted by Matson et al. (2012) comparing family reports from USA and South Korea they found that there were differences in the adaptive social skills exhibited by individuals with autism in the USA compared to South Korea. Although both these studies are multi-national, they fail to focus on the cultural differences existing within the population of each of these countries, some of which have substantial proportions of families from minority ethnic communities. It can also be argued that they found differences in the second study as they focused on two countries which enabled them to look for specifics.

Focusing on the implications of working with individuals on the spectrum, Dyches et al. (2007) and Lee (2011) highlight the importance of having social interaction goals that are appropriate to the child and its culture. However, before this is achieved, it is vital to understand what the cultural norms are for a given community and which skills are valued by parents from various communities. Unfortunately, there is still paucity of research exploring perceptions of parents from minority and majority ethnic groups living in the same country regarding their views on behaviours which are considered to be features of autism.

\section{Research aim}

This research tried to address this gap by investigating the cultural influence on the understanding of what constitutes appropriate social behaviour by parents of children with ASD, belonging to four different ethnic backgrounds, living in the United 
Kingdom. The four groups involved in the study were: White British, Somali, South Asian (India, Pakistan, and Bangladesh), and Afro-Caribbean (countries such as Nigeria, Ghana, Guyana, Congo and Sierra Leonia). There is no suggestion that these together form a homogenous group; however, as the members do share some cultural similarities, these categories are being used in an attempt to showcase the differences of opinions amongst the parents from each of these groups and initiate a dialogue regarding the universality of the concept of autism.

The main research questions were as follows;

(1) Are there cultural differences in the importance given to the different social behaviours found in ASD by the parents from these four communities?

(2) Which of the commonly associated social 'deficits' in autism do these parents consider as important skills to be taught to their children?

(3) What are the reasons which influence parents' selection of these skills?

\section{Methodology}

To address these research questions a qualitative approach was used as it allows the study of complex situations in their everyday context. This approach can also facilitate understanding the knowledge and practices of the participants (Flick, 2002), such as parental perceptions about social behaviour. Interpretive approaches believe that behaviours are evolving and are affected by the context (Cohen, Manion, and Morrison, 2011). This would mean that there could be multiple interpretations of the same situation, and that makes it difficult to generalise the findings. However, Denscombe (2003) argues that these multiple realities are based less on individual experiences than on the level of a group, culture, and society. On the basis of this 
argument, the findings from this kind of research can still be used to understand a group or culture. However, the intent of the study was not to generalise the findings but to provide a picture of the current scenario and the range of views held by parents from the four identified ethnic communities.

Purposive sampling was used to select participants from these four ethnic communities, living in an inner city in the UK both for the pilot study as well as the main research. Initially 'snowball' sampling was tried, but this was not effective as some parents were not aware of anyone else who had a child with ASD. This was especially the case with Somali participants, and so ASD support groups in the local area were contacted, some of whom included the information in their newsletters and others allowed attending their meetings to personally recruit the participants. The sample that emerged was therefore the result of these numerous recruitment approaches, and involved broadening the definition of the ethnic groups as described above. Table 1 shows that the number of participants in each group varied totalling to 29 White British, 16 Afro-Caribbean, 12 South Asian and 6 Somali. The proportion of fathers and mothers also varied in each group. This was a reflection of the difficulties which the project faced in recruiting participants.

\section{Table 1 Sample size here}

\section{Methods}

Semi-structured interviews were used for data collection because of their flexibility. This was an important element as the research included parents with range of competency in English. As this was a small-scale non-funded research, it was not 
possible to employ interpreters and therefore, providing explanations, and simplifying the language, was essential to gather the required data from these parents. The interview structure was based on an 'episodic interview' (Flick, 2002), which uses a combination of episodic narratives, along with confirming semantic knowledge. During the process of the interview the interviewee is periodically requested to narrate specific situations and is also asked for opinions and definitions. This latter process provides insight into the semantic knowledge of the interviewee. Flick (2002) considers that this approach has the advantages of both narrative and semi-structured interviews as the approach allows the interviewee to have an open dialogue and still provides analysable data about everyday events. This approach was used to gather information about how parents define social behaviour, and whether there are differences in understanding on the basis of ethnicity.

Providing an opportunity to define social behaviour (semantic knowledge) during the interview helped to clarify that most of the parents in the research understand social behaviour in the same way, i.e., how an individual interacts with others.

Establishing that there is a shared understanding then helped in the analysis stage to find out whether the differences were based on cultural factors. The narration of the episodes provided with examples where the parents considered a specific behaviour to be appropriate or inappropriate. Most of the interviews were recorded in an audio format and permission was taken from the participants before recording the interview. In total, thirteen out of the sixty-three interviews were not audio recorded due to lack of permission or environmental factors such as noise levels.

\section{Developing the interview guide}

The interview guide was developed from the outcomes of an initial focus group, 
a literature review of other research in the field, and the screening and diagnostic tools used in the field of ASD. The guide thus developed was piloted with a few parents which led to further refinement of the questions. To capture some of the variations between ethnic groups and to measure acculturation, an adapted version of the acculturation scale developed by Burnam et al. (1987) was used. In this scale Burnam et al. focused on three specific areas: the generation of the immigrants, the language they prefer to use, and the ethnicity of the people with whom they socialise. All these three areas were used in the interview guide although some of the wordings and situations referred to were changed. For example, while Burnam et al.’s scale only asks about language usage, the question in the interview guide for this research specifically asked for the language used at home. This was changed as it was felt the usage of language at home was a better measure of acculturation than usage of language in social settings. A question asking the individual to define their ethnicity was added, with the idea that this will be another indicator of how the participants perceive themselves rather than imposing an ethnic identity based on colour of skin or country of origin. These were used as some of the initial questions in the interview before the participants' views on autism and social behaviours were explored. Although this can be considered as a crude way of capturing information regarding acculturation this provided some insight into the cultural identity of the individual which was valuable during analysis stage.

Four common diagnostic and screening tools were selected to identify the common social behaviours associated with ASD. The tools used were: The Childhood Autism Rating Scale (Schopler, Reichler, \& Renner, 2002), Asperger Syndrome Diagnostic Scale (Myles, Bock, \& Simpson, 2001), Autism Diagnostic Interview- 
Revised (Rutter, Le Couteur, \& Lord, 2005), and Autism Diagnostic Observation Schedule (Lord, Rutter, DiLavore, \& Risi, 2003). After comparing the behaviours that occurred in all these tools, six social behaviours were identified to be included in the interview guide. These diagnostic tools were used because as Shakespeare and Watson (2001) suggest, disability has to be understood as a product of biological, psychological, cultural and socio-political factors. As these tools and characteristics are used for diagnostic purposes, it can be argued that they represent the socio-cultural interpretation of autism amongst the professionals in the west and would have been used to explain autism to parents irrespective of the age, gender or cultural background of the individual. The participants were requested to rate these six behaviours on the basis of the importance they assigned to each of them. The six identified behaviours were;

- using eye contact to communicate with adults and peers;

- using a range of facial expressions to express emotions;

- using gestures (including pointing);

- interacting with adults and people in authority- initiating conversations, requesting, commenting; 
- respecting personal space;

- following social rules, cues and boundaries for different situations.

Along with these, an open question was included requesting the interviewees to add two more behaviours that they consider as important, which were not covered in the list.

\section{Interview analysis}

Data thus generated was thematically analysed after all the audio-taped interviews were fully transcribed. This process provided an opportunity to get familiar with the data and in identifying the common themes. The transcribed interviews were then saved in an NVivo software package, which was used for coding and analysis purposes. NVivo was specifically selected because even after the interviews were coded, the information about the source and location of a quote is always preserved in NVivo, and it was possible to refer to the original context of the coded passages if clarification was required. Passages can also be coded at multiple 'nodes', as they were in this study, which could then be searched.

Thematic coding was applied as a multiple stage procedure, with the first step involving the interpretation of individual cases. Themes were then developed on the basis of this analysis, and these were then cross-checked and compared with other cases, thus enabling a comparison between the individuals and the collective understanding. 
Some of the categories which did not come up again in the other interviews were merged with a broader theme as sub-categories. Four sets representing the different ethnic groups were formed in NVivo, and interviews related to parents from each community were grouped under these sets. This then allowed for comparing the codes generated in each individual interview with other participants from the same ethnic background as well as with the other groups. This process of combining the experiences of individual participants into different ethnic groups gave an overview about the experiences of the community as a whole.

\section{Results and discussion}

The parents were asked to prioritise the six social behaviours. Some chose not to select certain behaviour, while others rated more than one at the same level as they considered all of them to be equally important. The first three ratings for each of the behaviours were totalled to get the aggregate percentages shown in Table 2. Parents were further asked to provide reasons for their choices.

\section{Table 2 here}

\section{What parents consider important to be taught}

There was no general agreement across the groups of parents as to whether all identified six social behaviours should be taught to children with ASD (see table 2). Where there were similarities in the importance attributed to particular behaviours, broadly speaking these were shared between the Afro-Caribbean and Somali groups, and between the South Asian and White British groups. The Afro-Caribbean and Somali parents considered giving eye contact as the most important skill. The South Asian and 
White British parents considered following rules and respecting personal space the most important ones. This difference in percentages for the six behaviours answers the first two research questions and suggests that there are cultural variations in the importance given to these behaviours and that parents from these cultural groups seem to prioritise different behaviours for their children on the autism spectrum. Having established this, the rest of this section focuses on the third research question and explores the reasons for these differences in opinion.

A number of themes emerged during the analysis of the data for this question such as 'developmental stages', 'deficits in autism',' better future', 'not appropriate for our culture',' characteristic of autism', 'models of disability' and 'issues of being a minority’. In this article, instead of focusing on each individual theme, those that directly answer the research question have been merged to provide an overview of the findings. As seen in Table 3, culture is treated as one main theme which includes subthemes of parent's gender, models of disability, important for Western society and acculturation. A number of non-cultural related sub-themes such as the age of the child, severity of their autism, and the training attended by the parent have been presented under another theme called 'other factors'.

\section{Table 3 here}

\section{Cultural differences}

The reasons most parents gave for teaching a particular behaviour were similar: to help their children to interact with others, to enable them to integrate into society, and a hope that learning these skills would help them to have a better future. Within this 
broad agreement, patterns of difference emerged based on culture and gender. For example, while White British parents felt facial expressions had limited importance in communication, parents from the other three groups felt that by learning this, their child would develop an understanding of others' emotions. As this South Asian mother states;

'Using facial expressions is second important to me. That will make him learn and be aware of others' emotions. I think the difference between humans and animals is that we are aware of others' emotions.'

Not many of the parents in this study specifically pointed towards cultural differences, but as seen in the above example, the fact that facial expressions have been considered to be more important by families from three ethnic groups compared to the White British group, could indicate that there is a cultural bias in the selection of these skills. Similar differences in the important associated can be seen for the other skills as well.

\section{Parents' gender:}

Across all the cultural groups, more fathers than mothers considered using a range of facial expressions to be a difficult skill to teach. This gender-based difference in the importance given to the skills was particularly evident in teaching to respect personal space, where a greater number of fathers than mothers considered this as an essential skill to teach their children. Similar cultural and gender differences have been found by previous researchers (Sue and Sue, 1990; Park, 1996) with regard to eye contact and personal space in anthropological studies. Gender rather than ethnic background seems to be more important for certain social behaviours. This needs to be considered while selecting social skills to teach. 


\section{Models of disability}

On the basis of the reasons the parents from the four ethnic groups gave for selecting some of the social behaviours it can be inferred that they are using different models of disability. For example, some of the parents from the three minority ethnic groups said that the reason they were selecting eye contact was that their child cannot do this, and it was a characteristic of ASD. This deficit model was not used as much by the White British parents for eye contact, with some of them using the social model and saying that society should make allowances. A White British mother argues this point in relation to eye contact,

'I started this at six... and the reason was because I think it is an over-rated skill and I know how hard autistic people can find it and I think they do it as much as they can do it and the rest of the time... I would rather he felt comfortable approaching me than actually looking at me and in terms of importance for social interaction in the future, I think making lots of eye contact is not that important...'

Some White British parents, however, used the deficit model as the reason for selecting skills such as respecting personal space, following social rules, using gestures, and interacting with adults, where some parents from the non-White backgrounds did not use it. This indicates that the views held by the parents were influenced to certain extent by the general debates taking place in the community about the appropriateness of teaching various skills, and the personal narratives of individuals on the spectrum. Access to these sources and literacy levels of the parents could be influencing their choice of skills. 


\section{Important in Western society}

Parents from minority ethnic communities felt that certain behaviours that are considered as inappropriate in their own culture were essential to learn because their child has ASD or to be able to live in the UK. For example, a Somali mother rated 'using gestures' as important for her son even though it is considered inappropriate in her own community. Similarly, this South Asian father uses his son's disability as the reason for selecting eye contact to be taught.

'Because I think it is a condition, Asperger's, where eye contact is important so I think, I am not going to go into that except for mentioning, I think eye contact is a cultural thing as well, but I am ignoring that cultural part of it. I am sticking to that that person has a disability and for him it is important to have eye contact so cultural issues shouldn't be there now.'

Another South Asian father also selected eye contact because it is important in Western society, as he explains,

'Even some socially active persons can sometimes feel like ...especially people coming from Bangladesh, because it has been taught that, if you look at the adult when you are speaking to them, you just think they are rude, too confident.... But in this country, people do not trust you if you do not look at them. They think the reverse in that case, that you could be lying. In our country it is politeness and in this country, in this culture, it could be lying, because you are not even looking at someone so that means you are probably not telling the truth. You are probably feeling embarrassed to look at the person's eyes.' 
It could be that parents coming from some minority ethnic communities are less confident in specifying their preferences because of their minority status in the society. Another possible reason for this difference could be as Parette, Chuang and Huer (2004) suggest, that acculturation within Western society influenced the way parents valued a set of skills.

\section{Influence of acculturation within British society}

While it is generally understood that acculturation consists in accepting the norms of the majority culture, it also includes developing the confidence to assert one's own culture. Both these strands were seen in the context of selecting skills such as eye contact, where some parents selected it because they thought it was an important skill to learn to live in the UK, while a third generation Caribbean mother thought it would be culturally inappropriate to teach it to her son.

The level of confidence can also be seen when groups as a whole are compared to each other. It was mainly the South Asian and the White British parents who said that some of the behaviours were inappropriate or unimportant to teach; by contrast, none of the Somali parents said this. This, to a certain extent, reflects the length of time that the different communities have lived in the UK. Out of the three minority ethnic groups, it was mainly the Afro-Caribbean and the South Asian parents in this study who have lived in the UK for longest, while most of the Somali parents were new immigrants. A similar pattern was followed when parents were asked if they would like to add any other skills to the list; it was mainly the White British and the South Asian groups that suggested additional skills. 
Sotnik and Jezewski (2005) describe acculturation as a multi-linear phenomenon which can be influenced by a number of factors including social class, education and gender. Social class and education were not specifically explored in this study and could be the contributory factors. However, the different emerging stages of acculturation were evident in the contradictory views that were held by some of the parents. For example, some Somali parents said that using gestures was an important skill to learn for non-verbal children with ASD. When they were asked later if learning any of the skills would cause problems in the social integration of their child within their community, some of these mothers also accepted that pointing was not considered appropriate by some members of their community, and therefore they would have to explain to them why their child was being encouraged to learn this. Most of the Somali mothers in this study had little education and came from lower socio-economic backgrounds.

\section{Other factors influencing the parents' perceptions}

It would be simplistic to suggest that differences noticed in this study can be solely attributed to a cultural basis. In addition to the influence of the gender of the parents, the age of the child, the severity of his/her ASD, and the training attended by the parents also seem to affect the importance they associate with the various social behaviours. For example, parents from Afro-Caribbean, White British, and South Asian groups felt that teaching about personal space is more important for older children and adults than younger children. A White British mother uses the same reason to explain the importance of following social rules and cues, 
'My son is very verbal, extremely verbal. He does not understand at all what different situations are and his inability to understand social situations means that he puts himself in danger. At the moment, particularly in terms of with teenagers, they can be pretty rude, swearing in public, and he is going to run up to them right in the middle and tell them off for swearing. He puts himself in danger and that limits any possible independence he could have, at the moment. '

Some Somali and White British parents selected teaching to use gestures because they thought it was an important skill for young children, and non-verbal children to learn. There were also disparities in the case of other social behaviours. Similarly, parents who had used Applied Behaviour Analysis with their children, or attended training in speech and language-based programmes preferred to use a developmental approach to teaching skills. Some parents also selected skills on their understanding of whether they were easy or difficult to teach. These additional factors support Gunaratnam's (2003) argument that culture cannot be studied in isolation and that other factors need to be considered.

\section{Implications of the study}

The evidence gathered in this research indicates that the importance given to the 'deficit' social behaviours associated with ASD is not universal but a social construct and is influenced by a number of factors including culture. This raises questions about the universality of the social skill training programmes in different cultures, or for different age groups and genders and indeed about the diagnostic criteria of autism itself. The difference in the importance attached to the social behaviours by individual 
parents needs to be considered when explaining the diagnosis of ASD to the parents as they may not consider the lack of certain skills as a disability but see it rather as an imposed label.

The skills that the parents selected to teach followed group patterns, but there were also differences on the basis of the gender of the parent as discussed in the results. This raises questions about validity of information being collected from one of the parents for the purpose of diagnosis, or putting interventions in place. It is possible that based on their perceptions about the difficulties of their child, the support needs of the parents themselves might be different. It is crucial to involve both parents when developing an intervention and selecting behaviours which need to be taught. It is also necessary to find out the value that the behaviour holds for the family concerned. Factors such as the age and the ability of the child can influence the parents' selection of skills. These differences in expectations need to be considered while providing intervention strategies for individuals on the autism spectrum and their families. If the teachers and other professionals are not engaged in such a dialogue it is possible that the parents will not follow the strategies.

\section{Limitations of the study}

The sample size and the varying numbers in each group is an important limitation of this study, but it does indicate the difficulty in engaging in a study like this, and the issue of recruiting participants, which required the re-definition of the ethnic groups during data gathering. Because of such broad definitions of communities, some of these groups actually included a number of different ethnicities, and this should be 
borne in mind when looking at the results of this research. Differences on the basis of the gender of the parent were also noticed in this study, and the results could have been different had equal proportions of men been recruited in all groups.

Lack of interpretation options could have hindered the participation of some parents and the depth of information provided by some others. Another weakness of this research was that it only focused on parental perceptions and not of those of the young people. Since findings from other studies suggest sense of identity in terms of disability and ethnicity vary between parents and the young people themselves (Atkin and Hussain, 2003) further research exploring these opinions in the field of autism is required.

\section{Conclusion}

This study was developed to explore parental attitudes towards social behaviours, and the value given to teaching some of these to their children with autism, on the basis of their cultural background. The findings from this study show parents cultural background along with other factors impacts the importance associated to the social behaviours related with autism spectrum. This could mean that behaviours which are considered appropriate by the teachers and other professionals within the school setting may not be expected within the child's home setting. Therefore, this study supports the argument made by Dyches et al. (2007) that it is important to consider the cultural appropriateness of the social skills being taught to children on the autism spectrum. Any impairment and its impact can only be understood within the context of the individual's experience of cultural and social networks. If cultural background 
influences which behaviours are considered problematic then this raises questions about how autism, which is after all diagnosed on the basis of observed behaviours, should be defined and indeed by whom. In fact should some of these characteristics associated with autism be considered as signs of impairment within those cultures?

This study has raised a number of questions that require further investigation to increase our knowledge about the understanding of autism in different cultures and to contribute to theorising differences. This is clearly a preliminary study, contributing to an area where there has been very little research until now, and upon which more robust replications can build. It is hoped that these findings might influence further research that will in turn have an impact on various stages of service delivery such as diagnosis, providing parental support, teaching appropriate social skills, and providing a range of culturally appropriate services, but most importantly lead to redefining the notion of autism. 


\section{Reference}

Allison, C., S. Baron-Cohen, S. Whhelwright, T. Charman, J. Richler, G. Pasco and C. Brayne. 2008. The Q-CHAT (Quantitative Checklist For Autism In Toddlers): A Normally Distributed Quantitative Measure of Autistic Traits at 18-24 Months of Age: Preliminary Report. Journal of Autism and Developmental Disorders, 38 (8): 1414-1425

Atkin, K. and Y. Hussain,. 2003. "Disability and Ethnicity: How Young Asian Disabled People Make Sense of Their Lives.” In Disability, Culture and Identity. edited by S. Riddell, and N. Watson. Harlow: Pearson

Burnam, M. A., R. L. Haugh, M. Karno, J. I. Escobar, and C. A. Telles. 1987.

“Acculturation and Lifetime Prevalence of Psychiatric Disorders among Mexican Americans in Los Angels”, Journal of Health and Social Behaviour 28: 89-102

Burton, M., C. Kagan, and P. Clements .1995. Social Skills for People with Learning Disabilities. London: Chapman and Hall

Charman, T. and W. Stone. eds. 2006. Social and Communication Development in Autism Spectrum Disorders. New York: The Guildford Press

Cohen, L., L. Manion, and K. Morrison. 2011. Research Methods in Education. $7^{\text {th }}$ ed. Abingdon: Routledge 
Daley, T. C. 2004. "From Symptom Recognition to Diagnosis: Children with Autism in Urban India.” Social Science and Medicine. 58: 1323-1335

Denscombe, M. 2003. The Good Research Guide. $2^{\text {nd }}$ ed. Berkshire: Open University

Dyches, T. T., K. Hobbs, L. K. Wilder, R. R. Sudweeks, F. E. Obiakor, and B. Algozzine. 2005. “Multicultural Representation in Autism.” Linking Research and Practice in Special Education- An International Perspective. 1(1): 1-15

Dyches, T. T., L. K. Wilder, B. Algozzine, and F. E. Obiakor. 2007. "Working with Multicultural Learners with Autism.” In F. E. Obiakor .ed. Multicultural Special Education: Culturally Responsive Teaching. New Jersey: Pearson

Flick, U. 2002. An Introduction to Qualitative Research, $2^{\text {nd }}$ ed. London: Sage

Fletcher, T. V., and L. A. Navarrete .2003. “Learning Disabilities or Difference: A Critical Look at Issues Associated with the Misidentification and Placement of Hispanic Students in Special Education Program.” Rural Special Education Quarterly. 22 (4): 3746

Fombonne, E., H. Simmons, T. Ford, H. Meltzer, and R. Goodman. 2001. "Prevalence of Developmental Disorders in the British Nationwide Survey of Child Mental Health.” Journal of the American Academy of Child and Adolescent Psychiatry. 40 (7): 820-827 
Gabel, S. 2004. "South Asian Indian Cultural Orientation Towards Mental Retardation.” Mental Retardation. 42(1): 12-25

Gunaratnam, Y. 2003. Researching Race and Ethnicity. London: Sage

Hatton, C. 2004. “Cultural Issues.” In The International Handbook of Applied Research in Intellectual Disabilities. edited by E. Emerson, C. Hatton, T. Thompson, and T. R. Parmenter. Chichester: John Wiley \& Sons

Kai, J., R. Loudon, and J. Beavan, .2006. “The Nature of Culture- Valuing Diversity and the Individual.” In Valuing diversity. edited by J. Kai. $2^{\text {nd }}$ ed. London: Royal College of General Practitioners

Kalyanpur, M. and B. Harry. 2004. "Impact Of The Social Construct Of LD On Culturally Diverse Families.” Journal of Learning Disabilities. 37(6): 530-533

Kim, W. J., L. I. Kim, and D. S. Rue. 1997. “Korean American Children.” In Transcultural Child Development- Psychological Assessment and Treatment. edited by G. Johnson-Powell and J. Yamamoto. New York: John Wiley and sons

Lee, H. J. 2011. “Cultural Factors Related to the Hidden Curriculum for Students with Autism and Related Disabilities.” Intervention in School and Clinic. 46(3):141-149

Lindsay, G., S. Pather, and S. Strand. 2006. Special Educational Needs and Ethnicity: Issues of Over and Under-Representation. Nottingham: DfES 
Liu, G. Z. 2005. “Best Practices- Developing Cross-Cultural Competence from Chinese Perspective.” In Culture and disability. edited by J. H. Stone. California: Sage

Lord, C., M. Rutter, P. C. DiLavore, and S. Risi. 2003. Autism Diagnostic Observation Schedule. Los Angeles: Western Psychological Services

Matson, J. L., J. A. Worley, J. C. Fodstad, K. M. Chung, D. Suh, H. Kyung Jhin, E. Ben-Itzchak, D. A. Zachor, and F. Furniss. 2011. “A Multinational Study Examining the Cross Cultural Differences in Reported Symptoms of Autism Spectrum Disorders: Israel, South Korea, the United Kingdom, and the United States of America.” Research in Autism Spectrum Disorders. 5: 1598-1604

Matson, J. L., J. A. Worley, A. M. Kozlowski, K. Chung, W. Jung, and J. Yang. 2012. “Cross Cultural Differences of Parent Reported Social Skills in Children with Autistic Disorder: An Examination between South Korea and the United States of America.” Research in Autism Spectrum Disorders. 6: 971-977

Myles, B.S., S. J. Bock, and R. L. Simpson. 2001. Asperger Syndrome Diagnostic Scale. Texas: Pro-Ed

Parette, P., S. Chuang, M. B. Huer. 2004. “First-Generation Chinese American Families’ Attitudes Regarding Disabilities and Educational Interventions.” Focus on Autism and other Developmental Disabilities. 19 (2): 114- 123 
Park, H. 1996. “Korean-American Families of Children with Disabilities; Perspectives and Implications for Practitioners.” In Teaching Students with Moderate/ Severe Disabilities, Including Autism. edited by E. Duran. $2^{\text {nd }}$ ed. Springfield: Charles Thomas

Perepa, P. 2013. "Implications of Special Needs and Multiculturalism for the Early Years Curriculum.” In The Early Years Curriculum: The UK Context and Beyond edited by L. Ang. Abingdon: Routledge

Rutter, M., A. Le Couteur, and C. Lord. 2005. Autism Diagnostic Interview- Revised. Los Angeles: Western Psychological Services

Schopler, E., R. J. Reichler, and B. R. Renner. 2002. The Childhood Autism Rating Scale. Los Angeles: Western Psychological Services

Shakespeare, T., and N. Watson. 2001. "The Social Model of Disability: An Outdated Ideology? In Exploring Theories and Expanding Methodologies: Where we are and Where we Need to go.” Research in Social Science and Disability. 2: 9-28

Sotnik, P. and M. A. Jezewski. 2005. “Culture and the Disability Services.” In Culture and Disability. edited by J. H. Stone. California: Sage

Sue, D. W., and D. Sue. 1990 Counselling the Culturally Different: Theory and Practice. $2^{\text {nd }}$ ed. New York: Wiley 
Wilder, L. K., T. T. Dyches, F. E. Obiakor, and B. Algozzine. 2004. "Multicultural Perspectives on Teaching Students with Autism.” Focus on Autism and other Developmental Disabilities. 19 (2): 105- 113

Wing, L. 1988. “The Continuum of Autistic Characteristics.” In Diagnosis and Assessment in Autism. edited by E. Schopler, and G. Mesibov. New York: Plenum Zionts, L. T., P. Zionts, S. Harrison, and O. Bellinger. 2003. "Urban African American Families Perception of Cultural Sensitivity within the Special Education System.” Focus on Autism and other Developmental Disabilities. 18(1): 41-50 
Table 1. Sample size

\begin{tabular}{|c|c|c|c|c|}
\hline Ethnicity & \begin{tabular}{|l} 
White \\
British
\end{tabular} & Afro-Caribbean & South Asian & Somali \\
\hline Fathers & 9 & 3 & 5 & 0 \\
\hline Mothers & 20 & 13 & 7 & 6 \\
\hline Total & 29 & 16 & 12 & 6 \\
\hline
\end{tabular}


Table 2. Social behaviours to be taught

- Using eye contact to communicate with adults and peers

Somali (83\%), Afro-Caribbean (67\%), South Asian (50\%), White British (32\%)

- Using a range of facial expressions to express emotions

Afro-Caribbean (53\%), Somali (50\%), White British (20\%), South Asian (16\%)

- Using gestures (including pointing)

Somali (67\%), White British (43\%), South Asian (41\%), Afro-Caribbean (39\%)

- Interacting with adults and people in authority- initiating, conversations, requesting, commenting

Somali (83\%), South Asian (74\%), White British (72\%), Afro-Caribbean (39\%)

- Respecting personal space

South Asian (67\%), White British (54\%), Afro-Caribbean (46\%), Somali (16\%)

- Following social rules, cues and boundaries for different situations

White British (93\%), South Asian (75\%), Somali (67\%), Afro-Caribbean (60\%) 
Table 3.Themes and sub-themes

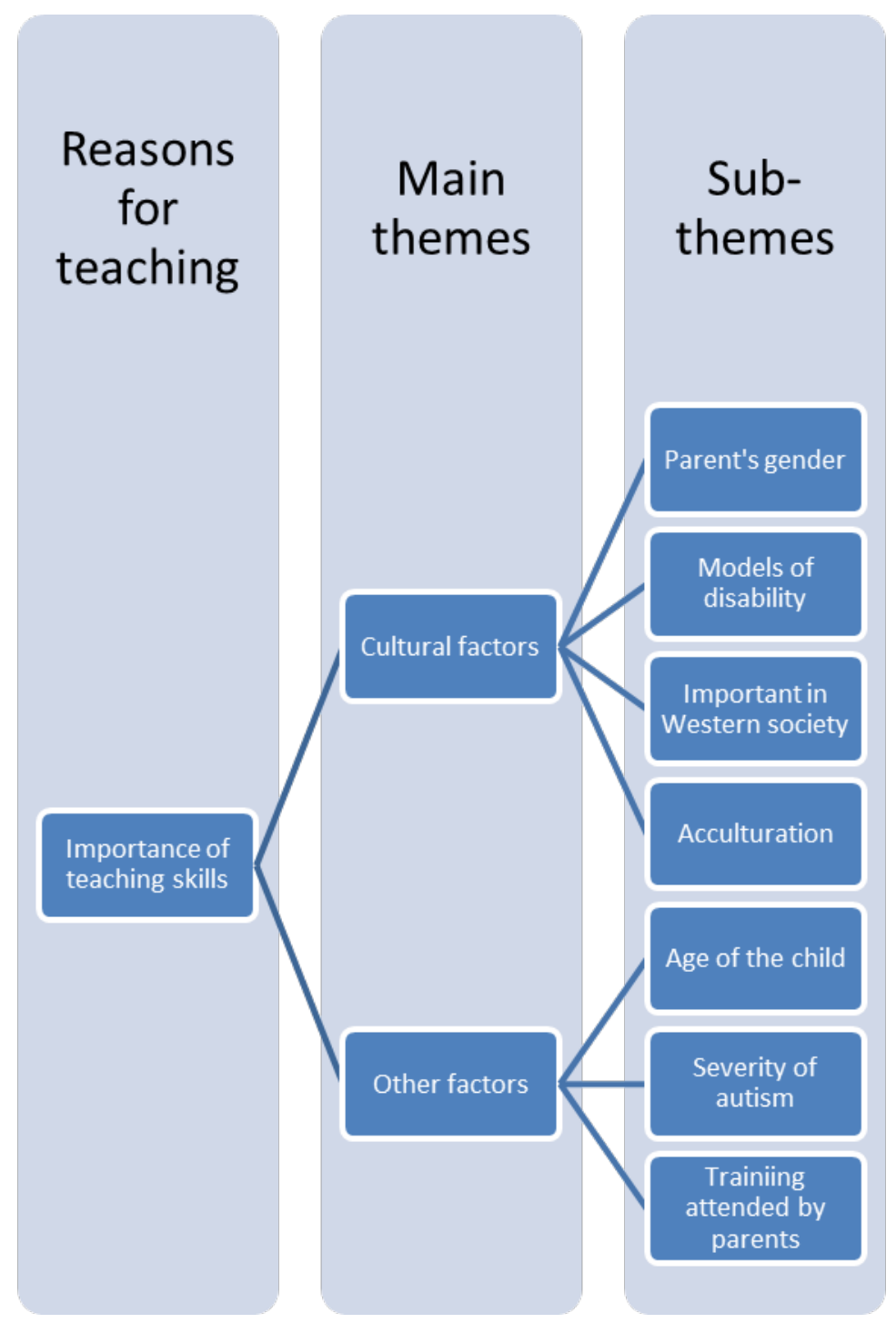

